\title{
Tamanho médio de partícula da dieta: determinação, interpretação e efeitos para vacas leiteiras
}

\author{
Average particle size of the diet: determination, interpretation and \\ effects for dairy cows
}

Daniel Augusto Barreta (10*

Universidade do Estado de Santa Catarina (Udesc), Chapecó, SC, Brasil

\section{Resumo}

A fibra é um componente do alimento essencial para os ruminantes. Sua efetividade em promover a ruminação e salivação é fundamental para manutenção da saúde dos animais. Contudo, além da concentração de fibra em detergente neutro (FDN), outras características físico-químicas dos alimentos são importantes para determinar a FDN fisicamente efetiva (FDNfe) da dieta, principalmente o tamanho da partícula. Neste sentido, é importante atentar-se à evolução do manuseio e interpretação dos resultados do conjunto de peneiras chamado Penn State Particle Separator, principal ferramenta prática utilizada para determinar o tamanho médio de partícula (TMP). A partir dessas constatações é possível implementar procedimentos que adequem o FDNfe das dietas e os processos de ensilagem. Estes ajustes podem atenuar problemas como a acidose ruminal, depressão da gordura do leite, seleção da dieta pelos animais, limitação física de ingestão, má compactação dos silos, entre outros. Desta forma, o objetivo desta revisão é realizar um apanhado evolutivo sobre os métodos para determinação do TMP e sua interpretação, além de discorrer sobre seus efeitos na nutrição de vacas leiteiras.

Palavras-chave: Acidose ruminal. Compactação. Fibra fisicamente efetiva. Penn State Particle Separator.

\begin{abstract}
Fiber is a component of essential food for ruminants. Its effectiveness in promoting rumination and salivation is fundamental to maintaining animal health. However, in addition to the neutral detergent fiber (NDF) concentration, other physical and chemical characteristics of the food are important in determining the physically effective NDF, especially particle size. In this sense, it is important to consider the evolution of the handling and
\end{abstract}


interpretation of the results of the set of sieves called the Penn State Particle Separator, the main practical tool used to determine the average particle size (APS). Based on this, it is possible to implement procedures that adapt the NDFpe of the diets and the ensilage processes. These adjustments can attenuate problems such as ruminal acidosis, milk fat depression, dietary selection of animals, physical limitation of feed intake, poor compaction of silos, and others. In this way, the objective of this review is to perform an evolutionary survey on the methods for determination of APS and its interpretation, in addition to discussing its effects on dairy cow nutrition.

Keywords: Ruminal acidosis. Compaction. Physically effective fiber. Penn State Particle Separator.

\section{Introdução}

Determinar a quantidade de fibra em uma dieta de forma que não limite a ingestão de matéria seca e que não cause efeitos colaterais à saúde animal é um desafio que acompanha os nutricionistas há bastante tempo, principalmente tratando-se de vacas leiteiras.

Contudo não apenas o conteúdo de fibra em detergente neutro (FDN) pode afetar a efetividade do material, como todas as variáveis físicoquímicas interagem entre si e podem afetar sua efetividade (Van Soest et al., 1991). Neste sentido, criou-se o conceito de fibra fisicamente efetiva (FDNfe), definido por Mertens (1994; 1997) como as propriedades físicas da porção fibrosa de um determinado alimento, capaz de promover a ruminação e mastigação, além de estratificar - conteúdo ruminal em duas frações: partículas maiores e flutuantes (mat ruminal) e partículas menores em emulsão.

De maneira geral, a efetividade física dos alimentos está relacionada à dimensão de suas partículas. O tamanho médio das partículas (TMP) é o principal fator que controla a taxa de passagem do rúmen, logo, o tempo de permanência no rúmen é um determinante de ingestão e digestibilidade (Welch, 1986). Não obstante, Mertens (1994) inferiu que a FDNfe de uma dieta é mensurada pela concentração de FDN contida nas frações retidas em uma peneira com orifícios de 1,18 $\mathrm{mm}$. Já partículas com dimensões menores saem do rúmen mais rapidamente e podem resultar na redução dos tempos de ruminação e decréscimo do $\mathrm{pH}$ ruminal (Ramirez et al., 2016).

Dietas à base de pasto ou com altas quantidades de forragem podem inibir que vacas leiteiras de alta produção consumam sua necessidade total de energia devido à limitação física provocada por forragens com teores de FDN elevados, as quais possuem menor digestibilidade e disponibilização de energia ao animal. Contudo, vacas leiteiras precisam ingerir quantidades mínimas de partículas longas provenientes de forragem para garantir a salivação, ruminação e motilidade ruminal, atividades que promovem a manutenção do $\mathrm{pH}$ e a saúde ruminal (Mertens, 1997; Allen, 2000).

Neste sentido, o National Research Council Dairy Cattle (NRC, 2001) recomenda que pelo menos $25 \%$ da dieta de vacas leiteiras seja composta por FDN, com no mínimo 19-21\% da FDN proveniente de forragem. Contudo estes parâmetros ainda estão sendo avaliados e variam de acordo com a produção animal, tipo de dieta, sistema de produção, entre outros. Além disso, é conveniente que as dietas não sejam formuladas com valores muito próximos aos limites estabelecidos pelo NRC (2001), pois os animais podem apresentar seletividade de consumo principalmente em dietas desuniformes e, com isto, consumir uma dieta com teores de fibra diferentes daquela fornecida (Campos, 2015).

Haja visto a importância da porção fisicamente efetiva da fibra para ruminantes e a relação direta com o tamanho médio de partícula, o objetivo desta revisão é realizar um apanhado sobre o método de determinação do TMP da Penn State University, sua interpretação e efeitos na nutrição de vacas leiteiras.

\section{Evolução da determinação do TMP}

O TMP é uma variável muito importante na nutrição de vacas leiteiras e tem influência direta na taxa de passagem da digesta, ingestão de matéria 
seca e saúde animal (Welch, 1986). Os principais alimentos que podem ser mensurados quanto ao TMP são: silagem, feno e pré-secado. Além disto, também é possível avaliar o TMP na dieta quando esta é fornecida na forma de dieta total misturada.

Os primeiros passos para a determinação da FDN fisicamente efetiva foram baseados em resultados prévios que definiram o tamanho de partícula limiar para promover a ruminação em 1,18 $\mathrm{mm}$, haja visto que menos de $5 \%$ das partículas que saíam do rúmen tinham diâmetro superior a este; logo, para determinar a fração de FDNfe seria necessário considerar o percentual de FDN em partículas maiores que 1,18 mm (Mertens, 1997).

$\mathrm{Na}$ mesma época, Lammers et al. (1996) buscaram desenvolver um método prático que quantificasse o tamanho médio de partículas. Esta necessidade surgiu devido à complexidade e difícil praticidade do método S424 proposto pela American Society of Agricultural Engineers (ASAE, 1993). Os pesquisadores desenvolveram o método Penn State Particle Separator (PSPS), cujo nome faz referência à Universidade Estadual da Pensilvânia (Pennsylvania State University). Tal método consistia no uso de duas peneiras seguidas de um fundo, com orifícios de 19 e $8 \mathrm{~mm}$. Após uma série de agitações, a estratificação servia como parâmetro para a análise de FDNfe.

Embora o modelo proposto tenha sido amplamente utilizado, observaram-se algumas limitações do método, principalmente quando utilizado para avaliar a dieta total, uma vez que na maioria dos casos a dieta contém uma grande proporção de concentrado, que facilmente passava através da peneira de $8,0 \mathrm{~mm}$. Neste sentido, foram recomendadas algumas adaptações, como a inclusão de uma terceira peneira com orifícios de 1,18 mm e padronização de agitação a uma frequência de 66 ciclos/ minuto, com um comprimento de curso de $17 \mathrm{~cm}$ (Kononoff et al., 2003).

Estudos mais recentes, entretanto, verificaram que o limiar crítico para a passagem de partículas pelo orifício retículo omasal é variável e maior que $1,18 \mathrm{~mm}$, sendo de pelo menos $4 \mathrm{~mm}$ para vacas leiteiras de alta produção (Maulfair et al., 2011). Neste sentido, a mais recente (Heinrichs e Jones, 2013) mudança da PSPS contempla um conjunto de três peneiras com orifícios de 19 e $8 \mathrm{~mm}$ de diâmetro, assim como o modelo anterior (Kononoff et al., 2003), porém a peneira de $1,18 \mathrm{~mm}$ foi substituída por uma com orifícios de $4 \mathrm{~mm}$. Estas peneiras separam frações distintas do alimento. A maior delas quantifica partículas grandes, que flutuariam no rúmen e promoveriam mastigação adicional, logo, favorecem a manutenção do pH pela salivação. As partículas retidas na peneira de $8 \mathrm{~mm}$ farão parte do mat ruminal e também podem estimular a mastigação, porém tendem a ser degradadas mais rapidamente pelos microorganismos do rúmen. Já a peneira de $4 \mathrm{~mm}$ retém partículas que são facilmente quebradas pela ruminação ou ação microbiana; mesmo assim, são significativas para determinar o FDNfe, ou seja, o foco está em uma distribuição equilibrada das partículas (Heinrichs e Jones, 2013).

O uso do PSPS também pode ser empregado para avaliar a seleção dos componentes da dieta pelos animais, bastando realizar aferições da dieta antes e após a alimentação. Em geral, os valores não podem variar mais do que $5 \%$. Outra aplicação prática do conjunto é a avaliação da qualidade do misturador da dieta total (TMR total mixed ration): uma mistura em demasia causa a redução do tamanho de partícula; por outro lado, uma submistura dificulta a homogeneidade de distribuição da dieta ao longo do comedouro (Park et al., 2015). As instruções completas do uso podem ser consultadas em Kononoff et al. (2003).

Neste mesmo escopo, Schuler et al. (2014) utilizaram o PSPS para determinar o tempo de mistura e a ordem de colocação dos ingredientes em vagões forrageiros. Para uma mistura que continha milho, feno e grãos de destilaria úmidos, o menor coeficiente de variação da distribuição das amostras no PSPS foi obtido com o tempo de mistura de 3 minutos vs. 5 e 7 minutos. Quanto à distribuição das partículas na peneira, Perricone et al. (2018) verificaram uma tendência de maior retenção $(14,79$ vs. $10,14 \%, p=0,06)$ de partículas na primeira peneira, com tempo de mistura menor que 7 minutos em comparação a misturas que duraram mais que 7 minutos. Os autores sugerem a avaliação recorrente da distribuição do tamanho de particulas, evitando, assim, oscilações na ingestão de matéria seca (IMS). 


\section{Coleta de amostras para determinação do TMP}

Como descrito acima, houve uma evolução constante da técnica de avaliação do tamanho médio de partícula dos alimentos fornecidos aos animais. Contudo, tão importante quanto o procedimento de análise é o método de coleta das amostras. Ao estudarmos determinada característica, normalmente buscamos gerar inferências capazes de serem aplicadas a toda a população. Como não temos acesso a todos os indivíduos, faz-se o uso de técnicas para amostragem dos materias com o intento de que aquela amostra seja representativa do todo (Piana et al., 2009).

De maneira geral, quanto mais subamostras forem utilizadas para compor uma amostra, maior será a representatividade amostral. Embora não se tenham metodologias específicas para a coleta de amostras, em geral as pesquisas tem empregado a metodologia tipo "W" ou "zig-zag" no perfil do silo para a coleta de amostras de silagem (StPierre e Weiss, 2015; Oliveira et al., 2017). Após a coleta das subamostras, o procedimento ideal é a homogeinização das amostras em superfície plana, seguida de um quarteamento. A partir deste ponto, opta-se por uma das frações, que deve ser embalada em recipiente plástico com o mínimo de oxigênio possível (Carvalho, 2011).

Para a coleta de TMR são necessárias algumas precauções, pois a dieta é composta de diversas partículas com tamanho e densidade diferentes. Weiss et al. (2016) verificaram resultados precisos para porcentagem de proteína bruta (PB), FDN e matéria seca (MS) de TMR a partir da amostragem a cada 3 metros de linha de cocho, alternando entre o terço superior médio e inferior do alimento (profundidade da amostragem). Os autores ainda reiteraram que se o amostrador utilizar diretamente a mão para realizar a coleta é importante que seja com a palma da mão para cima, a fim de evitar a perda de partículas pequenas, o que pode gerar um viés na amostragem.

Em relação ao feno, mesmo que o material seja oriundo do mesmo lote, pode haver diferenças consideráveis entre os fardos. Collins et al. (2000) verificaram uma variação entre 58 e $65 \%$ do teor de
FDN entre fardos de alfafa/gramíneas do mesmo lote. Os autores sugerem que sejam amostrados pelo menos 20 fardos por lote e, segundo Martin et al. (1988), essa amostragem deve ser realizada retirando-se uma fração do centro do fardo, afastado das laterais. O mesmo procedimento pode ser considerado para o feno pré-secado (haylage), com a retirada de uma amostra perpendicular à circunferência do fardo. Admitese, contudo, uma amostragem de menos fardos, devido à necessidade de abertura do plástico que envolve o material (Undersander et al., 2005).

\section{Interpretação do TMP}

Após uma correta amostragem do painel do silo, da dieta total dos animais ou ainda da silagem pré-emurchecida (comumente chamada de "silagem pré-secada" ou "feno pré-secado"), aplica-se a metodologia de avaliação do tamanho de partículas. É necessário interpretar com clareza os resultados a fim de determinar a porcentagem correspondente de cada amostra retida nas malhas em relação à amostra total.

As recomendações de distribuição das amostras nas peneiras foram determinadas por Heinrichs e Jones (2013). Segundo os autores, a silagem de milho retida na peneira superior deve ser entre 3 e $8 \%$, sendo o mínimo de $8 \%$ recomendado para situações na qual a silagem é a única fonte forrageira para os animais. Já valores próximos a 3\% são ideais para dietas com outras fontes de forragem. Espera-se que 45 a $65 \%$ do material permaneça sobre a peneira com crivo de $8 \mathrm{~mm}$, e entre 20 e $30 \%$ sobre a peneira com crivo de $4 \mathrm{~mm}$. Vale ressaltar que nesta última peneira há a possibilidade de retenção de grãos, os quais devem ser descartados, pois sua contribuição como fibra fisicamente efetiva é irrisória. Neste sentido, o recipiente de fundo não deve ter mais de $10 \%$ da amostra, isso porque a silagem de milho é essencialmente um volumoso, ou seja, precisa estimular a ruminação dos animais.

Em geral, as partículas de silagem devem ter tamanho médio de 1 a $2 \mathrm{~cm}$ para atender aos requisitos nutricionais que lhes são atribuídos. Além disto, em relação ao processo de confecção 
da silagem, a presença de partículas muito grandes tende a dificultar a compactação no silo e, com isso, predispor o material à degradação microbiana (Muck e Holmes, 2000).

Para silagem pré-emurchecida acondicionada em plástico filme ou silagem de forrageiras, os valores tidos como ideias são variáveis em função da forragem utilizada. Em média, a peneira superior deve reter uma fração entre 10 e $20 \%$, a peneira intermediária entre 45 a $75 \%$ (ou seja, as partículas devem ser homogêneas e eficientes em promover a ruminação e salivação do animal), a peneira inferior deve reter entre 30 e $40 \%$ e o fundo não mais do que 10\% (Heinrichs e Jones, 2013).

É importante ressaltar que é crescente no Brasil a adoção de sistemas de produção de leite nos quais os animais permanecem confinados, como o compost barn (Danieli et al., 2018), e que nestes sistemas é preponderante o fornecimento da dieta total misturada, procedimento que traz consigo benefícios como a facilidade de manejo e homogeneidade da dieta dos animais (quando bem misturada). Além disto, estes sistemas facilitam a determinação das necessidades nutricionais dos animais, dentre elas a necessidade de fibra fisicamente efetiva.

Dentro deste escopo, determinar o tamanho médio de partícula da dieta é uma ferramenta muito útil nas fazendas leiteiras. Como a variabilidade de produtos utilizados para compor uma dieta é alta, as recomendações são variáveis, contudo, admite-se até $8 \%$ do material retido na peneira superior, 30 a $50 \%$ na peneira intermediária, 10 a 20\% na peneira inferior e entre $30 \%$ e $40 \%$ no fundo (Heinrichs e Jones, 2013). É importante salientar que quando o objetivo é quantificar a fibra, grãos ou outros subprodutos que por ventura permaneçam retidos deverão ser desconsiderados.

\section{Uso do TMP para confecção de silagens}

A aplicação do método de determinação do TMP é uma ferramenta muito importante para produtores e técnicos; sua interpretação correta permite identificar fontes de problemas nutricionais e nortear soluções. Além disto, a interpretação dos resultados ainda na fase de confecção das silagens permite ao produtor a possibilidade de contornar os erros e adequar o procedimento.

Em termos de TMP de silagem de milho, os principais fatores que podem alterar esta medida são: o teor de matéria seca da planta, genótipo, uso do quebrador de grão, afiação das facas e distância entre estas, e velocidade de trabalho (Neumann et al., 2007; Marafon et al., 2015). Weirich Neto et al. (2013) também verificaram que o tamanho das partículas da silagem de milho em função da regulagem foi diferente daqueles indicados pelo fabricante de uma forrageira movida a trator, o que realça a importância da avaliação durante a colheita do TMP.

Avaliando a qualidade da silagem de milho produzida em fazendas leiteiras de alta produção no Brasil, Oliveira et al. (2017) coletaram amostras provenientes de quatro estados da federação e avaliaram diversos parâmetros, entre eles o tamanho médio de partículas de acordo com a metodologia descrita por Heinrichs e Kononoff (2002). O tamanho médio verificado foi de 1,27 $\pm 0,27 \mathrm{~cm}$, com variações de 0,93 a 1,83 cm entre as fazendas. Os autores consideraram o valor adequado, haja visto que a recomendação difundida atualmente é de partículas entre 1 e $2 \mathrm{~cm}$.

Além dos efeitos do TMP da silagem na nutrição animal, suas dimensões também influenciam a qualidade da própria silagem, pois partículas maiores dificultam a compactação da massa, que fica mais vulnerável à penetração de oxigênio e degradação aeróbica; ou seja, a densidade é um dos principais fatores determinantes da qualidade do volumoso (Jobim et al., 2007).

\section{Efeitos do TMP sobre a produção e qualidade do leite}

A ingestão de fibras está associada principalmente ao consumo de MS e à saúde ruminal. Além disso, há uma necessidade mínima de FDN para a produção de leite com adequado teor de gordura (NRC, 2001). Também é importante ressaltar que, embora pareça um contrassenso, o aumento do tamanho médio de partículas pode representar um menor consumo de fibra fisicamente efetiva em sistemas de alimentação com TMR, pois vacas 
alimentadas com dieta total misturada contendo partículas longas podem apresentar seleção de alimentos e exercer uma preferência por partículas menores e mais energéticas. Desta forma, este comportamento de seleção pode promover um consumo excessivo de carboidratos facilmente fermentáveis e um subconsumo dos carboidratos fisicamente efetivos (Miller-Cushon e DeVries, 2017).

Nasrollahi et al. (2015) realizaram uma metaanálise sobre o efeito do TMP no consumo de nutrientes, produção de leite e digestibilidade, tomando por base dados publicados entre 1998 e 2014. Os tratamentos dos 45 trabalhos utilizados na meta-análise continham entre 35 e $80 \%$ de forragem na dieta. Os autores verificaram que a diminuição do tamanho médio de partículas proporcionou o incremento da ingestão diária de matéria seca, fibra em detergente neutro e produção de leite. No entanto a digestibilidade da fibra em detergente neutro diminuiu com a redução do TMP e a produção corrigida para gordura não foi alterada.

Factori et al. (2014) avaliaram a digestibilidade in vitro e a degradabilidade in situ de silagem de dois híbridos de milho (grão duro e dentado), processados ou não, três estádios de maturação e três tamanhos de partícula (2, 7 e $11 \mathrm{~mm}$ ). Houve interação entre o híbrido dentado não processado e o tamanho de partícula, de modo que a degradabilidade (20h) diminuiu $1,4 \%$ à medida que aumenta-se $1 \mathrm{~mm} \circ$ tamanho da partícula. Os autores atrelaram os resultados ao maior aporte de energia fornecido pelo processamento indireto de grãos, o que permite maior ataque dos microrganismos à parte fibrosa e, consequentemente, maior degradação.

Neste intento, Coon et al. (2018) avaliaram 41 vacas holandesas multíparas dispostas em um sistema free stall com alimentação individual (uma vez ao dia) durante os primeiros 28 dias de lactação. Os animais foram divididos em dois lotes logo após o parto, e receberam dietas iguais em termos de composição. A diferença entre os tratamentos consistia no tamanho médio de partícula da palha de trigo, 2,54 cm para o tratamento "partícula curta" ( $n=21)$ e $5,08 \mathrm{~cm}$ para o "longa" ( $=20)$. É importante salientar que a palha de trigo contribuía apenas com $9 \%$ da MS da dieta. Os resultados evidenciaram que o tamanho da palha de trigo induziu o comportamento de seleção, ou seja, os animais que receberam a dieta longa conseguiram separar as partículas maiores ( $>19 \mathrm{~mm}$ ) e o consumo destas foi inferior. Não foram observadas diferenças em termos de produção e composição do leite, comportamento alimentar e $\mathrm{pH}$ médio analisados semanalmente. No entanto, quando analisados diariamente, os animais do tratamento longo apresentaram um $\mathrm{pH}$ do retículo-rúmen inferior nos primeiros dez dias. Os autores sugerem que a separação das partículas longas pode ter contribuído para um ambiente retículo-rúmen menos estável.

Os efeitos da adição de gordura e dois tamanhos de partículas sobre a depressão da gordura do leite de vacas holandesas foram avaliados por Ramirez et al. (2016). Os animais foram alocados em um delineamento de quadrado latino e receberam as seguintes dietas por 28 dias: $0 \%$ de óleo de milho + partícula forrageira curta; $0 \%$ de óleo de milho + partícula forrageira longa; $2 \%$ de óleo de milho + partícula forrageira curta; $2 \%$ de óleo de milho + partícula forrageira longa. A forragem utilizada foi o capim Timothy (Phleum pratense) triturado através de uma tela de $4,8 \mathrm{~mm}$ para fazer pellets ou cortados em moinho com tela de $7,62 \mathrm{~cm}$. Os resultados não elucidaram diferenças no consumo de MS, porém a produção de leite (corrigida para $3,5 \%$ gordura) foi superior para os tratamentos sem o uso do óleo (34,55 vs. 26,55 kg/dia). Esta grande variação é decorrente justamente da porcentagem de gordura do leite, que não variou entre os tratamentos sem a inclusão de óleo $(3,62 \%)$, mas que decresceu com a introdução deste $(2,27$ e $3,02 \%$ para tamanho de partícula curta e longa, respectivamente). Os autores concluíram que o óleo diminui a gordura do leite, no entanto, seu efeito foi atenuado quando associado a uma fibra longa. O tempo gasto diariamente para alimentação e ruminação também foi influenciado pelo tamanho da fibra, logo, a taxa de passagem seguiu o mesmo comportamento.

Kmicikewycz e Heinrichs (2015) avaliaram o efeito do tamanho de partículas da silagem sobre a produção e composição do leite, comportamento alimentar e características de rúmen de vacas holandesas de alta produção com acidose ruminal 
subaguda. $O$ experimento foi bifatorial com silagem com partículas curtas ou longas e com ou sem suplementação de feno. A indução da acidose ocorreu por meio da adição direta no rúmen de $4 \mathrm{~kg}$ de trigo moído. A silagem curta apresentava TMP de 6,64 mm e a longa, 11,65 mm. A inclusão de feno foi de $5,6 \%$ do valor de ingestão da MS. Todos os animais diminuíram o valor de $\mathrm{pH}$ do rúmen com a inclusão do trigo moído, contudo não foram verificadas diferenças em função do TMP. Em relação ao consumo, a ingestão de MS foi superior para os animais com o uso de silagem curta tanto antes quanto após a inclusão do trigo $(29,85$ vs. $26,7 \mathrm{~kg} / \mathrm{dia}$ e 31,4 vs. $28,03 \mathrm{~kg} / \mathrm{dia}$, respectivamente). A produção de leite corrigida ( $3,5 \%$ de gordura) foi maior para os animais que consumiram silagem curta suplementada com feno em relação aos demais tratamentos $(43,6$ vs. $40,8 \mathrm{~kg} / \mathrm{dia}$ ). Sob a ótica do comportamento alimentar, vacas sem a disponibilidade de feno foram eficientes em selecionar a dieta e ingerir proporcionalmente mais partículas maiores que 19 $\mathrm{mm}$ e menos partículas entre 4 e $8 \mathrm{~mm}$.

Dentro deste contexto, Maulfair e Heinrichs (2013) avaliaram a interação entre o tamanho de partícula forrageira e o teor de carboidratos degradáveis no rúmen. Os tratamentos do estudo foram tamanhos de partícula da silagem, longo e curto, associados com grão de milho quebrado e moído. AIMS foi menor para os animais alimentados com silagem longa e grãos quebrados em relação aos demais (27,9 vs. $31,2 \mathrm{~kg} / \mathrm{dia}$ ) e o pH ruminal foi semelhante entre os tratamentos. Já a produção de leite foi superior para os dois tratamentos com o uso do milho moído em relação aos dois tratamentos com uso de milho quebrado (45 vs. $41,9 \mathrm{~kg} / \mathrm{dia}$ ). Este resultado pode ser um efeito do aumento da energia disponível no rúmen para o tratamento moído, o que aumenta a síntese de proteína microbiana e a produção de propionato, ligado à produção de leite. Já o percentual de gordura no leite apresentou comportamento inverso: os animais que receberam milho quebrado na dieta tiveram maior porcentagem de gordura do que aqueles cujo milho era moído (3,54 vs. 3,34\%). Este resultado fez com que a produção de leite corrigida para 3,5\% de gordura fosse semelhante entre as quatro dietas.
No intuito de avaliar o efeito do TMP da dieta total dos animais, Maulfair et al. (2010) alimentaram vacas holandesas com quatro dietas idênticas, que apresentaram diferenças apenas no comprimento da partícula de feno, designadas como curta, média, longa e extralonga; com isso, o TMP das dietas foi respectivamente 4,$46 ; 5,10$; 5,32; 5,84 mm. Houve uma tendência linear de diminuição da IMS pelo aumento do TMP da dieta. Quanto ao comportamento alimentar, não houve diferenças entre as dietas para tempo ( $\mathrm{min} /$ dia) de ruminação, alimentação e mastigação. Já para as variáveis tempo gasto para ingestão de um $\mathrm{kg}$ de $\mathrm{MS}(\mathrm{min} / \mathrm{kg})$ e número de mastigações para ingestão de um kg de MS (mastigações $/ \mathrm{kg}$ ), houve um aumento linear no tempo e número de mastigações em função do aumento do tamanho médio das partículas da dieta. Apesar disso, o pH ruminal e a produção de leite foram semelhantes entre as dietas.

\section{Conclusão}

O conjunto de peneiras Penn State Particle Size foi ajustado ao longo do tempo à luz de descobertas da área de nutrição de ruminantes, associado à praticidade e padronização do manuseio. A recente inclusão da peneira com orifícios de 4 $\mathrm{mm}$, associada às peneiras de 8 e $19 \mathrm{~mm}$, permite uma melhor discriminação do tamanho médio das partículas e, por consequência, o ajuste do nível de fibra fisicamente efetiva para vacas leiteiras, além da adequação dos processos de ensilagem. A diminuição do tamanho médio de partículas na dieta de vacas leiteiras promove maior ingestão de matéria seca e menor taxa de mastigação e ruminação. Além disto, torna os animais menos eficazes em separar as partículas da dieta total misturada que, por sua vez, parecem habituar-se a uma constante acidose subaguda.

\section{Referências}

Allen MS. Effect of diet on short-term regulation of feed intake by lactating dairy cattle. J Dairy Sci. 2000;83(7):1598-624. 
ASAE - American Society of Agricultural Engineers. Standards S424. Method of Determining and Expressing Particle Size of Chopped Forage. 40 ed. St. Joseph, MI, USA: American Society of Agricultural Engineers; 1993.

Campos AF. Efeito do tamanho de partícula na ensilabilidade e no valor nutritivo de silagens de canade-açúcar para bovinos de corte [tese]. Jaboticabal: Universidade Estadual Paulista; 2015. 105 p.

Carvalho IQ. Metodologia para coleta de amostra de silagem. Fundação ABC: Setor de Forragicultura; 2011.

Collins M, Putnam D, Owens V, Wolf M. Hay Sampling Principles and Practices. 2000 [acesso 29 fev 2019]. Disponível em: https://tinyurl.com/r9z7by5.

Coon RE, Duffield TF, DeVries TJ. Effect of straw particle size on the behavior, health, and production of earlylactation dairy cows. J Dairy Sci. 2018;101(7):6375-87.

Danieli B, Barreta DA, Schogor ALB. Características e recomendações de gerenciamento no confinamento de vacas de leite em compost barn: revisão. Sci Agrar. 2018;19(2):249-55.

Factori MA, Costa C, Meirelles PRL, Silveira JPF, Silva MGB. Degradabilidade e digestibilidade de híbridos de milho em função do estádio de colheita, tamanho de partícula e processamento por meio do esmagamento na ensilagem. Biosci J. 2014;30(5 suppl. 2):882-91.

Heinrichs J, Kononoff P. Evaluating particle size of forages and TMRs using the new Penn State Forage Particle Separator (Technical Repost DAS 02-42). Pennsylvania, USA: The Pennsylvania State University/Department of Dairy and Animal Science; 2002. 14 p.

Heinrichs J, Jones CM. The Penn State Particle Separator. DSE 2013-186. 2013 [acesso 28 mai 2018]. Disponível em: https://tinyurl.com/rkzp4yf.

Jobim CC, Nussio LG, Reis RA, Schmidt P. Avanços metodológicos na avaliação da qualidade de forragem conservada. R Bras Zootec. 2007;36(supl. esp.):101-19.

Kmicikewycz AD, Heinrichs AJ. Effect of corn silage particle size and supplemental hay on rumen $\mathrm{pH}$ and feed preference by dairy cows fed high-starch diets. J Dairy Sci. 2015;98(1):373-85.

Kononoff PJ, Heinrichs AJ, Buckmaster DR. Modification of the Penn State forage and total mixed ration particle separator and the effects of moisture content on its measurements. J Dairy Sci. 2003;86(5):1858-63.

Lammers BP, Buckmaster DR, Heinrichs AJ. A Simple Method for the Analysis of Particle Sizes of Forage and Total Mixed Rations. J Dairy Sci. 1996;79(5):922-8.

Maulfair DD, Fustini M, Heinrichs AJ. Effect of varying total mixed ration particle size on rumen digesta and fecal particle size and digestibility in lactating dairy cows. J Dairy Sci. 2011;94(7):3527-36.

Maulfair DD, Heinrichs JA. Effects of varying forage particle size and fermentable carbohydrates on feed sorting, ruminal fermentation, and milk and component yields of dairy cows. J Dairy Sci. 2013;96(5):3085-97.

Maulfair DD, Zanton GI, Fustini M, Heinrichs AJ. Effect of feed sorting on chewing behavior, production, and rumen fermentation in lactating dairy cows. J Dairy Sci. 2010;93(10):4791-803.

Marafon F, Neumann M, Ribas TNB, Reinehr LL, Poczynek M, Bueno AVI, et al. Análise do efeito da colheita da planta de milho em diferentes estádios reprodutivos e do processamento dos grãos sobre a qualidade da silagem. Semina Cienc Agrar. 2015;36(5):3257-68.

Martin NP, Ellingboe RL, Linn JG. Hay sampling and testing. Proceedings 18th National Alfalfa Symposium; 2-3mar 1988; St. Joseph, MO, USA. St. Paul, MN, USA: Certified Alfalfa Seed Council;1988. p. 92-102.

Mertens DR. Creating a system for meeting the fiber requirements of dairy cows. J Dairy Sci. 1997;80(7):1463-81.

Mertens DR. Regulation of forage intake. In: Fahey JR et al. (EE.). Forage quality, evaluation and utilization. Wisconsin: ASA-CSSA-SSSA; 1994. p. 450-93.

Miller-Cushon EK, DeVries TJ. Feed sorting in dairy cattle: causes, consequences, and management. J Dairy Sci. 2017;100(5):4172-83. 
Muck RE, Holmes BJ. Factors affecting bunker silo densities. Appl Eng Agric. 2000;16(6):613-9.

Nasrollahi SM, Imani M, Zebeli Q. A meta-analysis and meta-regression of the effect of forage particle size, level, source, and preservation method on feed intake, nutrient digestibility, and performance in dairy cows. J Dairy Sci. 2015;98(12):8926-39.

Neumann M, Mühlbach PRF, Nörnberg JL, Restle J, Ost PR. Efeito do tamanho de partícula e da altura de colheita de plantas de milho (Zea mays L.) para ensilagem na produção do novilho superprecoce. R Bras Zootec. 2017;36(5):1614-23.

NRC. Nutrient requirements of dairy cattle. 7th Revised Edition. Washington, D.C.: Subcommittee on Dairy Cattle Nutrition, Committee on Animal Nutrition, Board on Agriculture and Natural Resources, National Research Council, National Academy Press; 2001.

Oliveira IL, Lima LM, Casagrande DR, Lara MAS, Bernardes TF. Nutritive value of corn silage from intensive dairy farms in Brazil. R Bras Zootec. 2017;46(6):494-501.

Park JH, Kim KH, Park PJ, Jeon BT, Oh MR, Jang SY et al. Effects of physically effective neutral detergent fibre content on dry-matter intake, digestibility and chewing activity in beef cattle fed total mixed ration. Anim Prod Sci. 2015;55(2):166-9.

Perricone V, Agazzi A, Costa A, Lazzari M, Savoini G, Calcante $A$, et al. Chemical homogeneity and particle size distribution of dairy cow TMR along the feeding alley with different mixing times. Int $\mathrm{J}$ Health Anim Sci Food Safety. 2018;5(1s):67-8.

Piana CFB, Machado AA, Selau LPR. Estatística Básica (versão preliminar). Pelotas: UFPel, Instituto de Física e Matemática; 2009. 220 p.
Ramirez HAR, Harvatine KJ, Kononoff PJ. Short communication: Forage particle size and fat intake affect rumen passage, the fatty acid profile of milk, and milk fat production in dairy cows consuming dried distillers grains with solubles. J. Dairy Sci. 2016;99(1):392-8.

Schuler NJ, Bern CJ, Loy DD, Brumm TJ, Strohbehn DR. Mixing beef feed rations containing distillers wet grains. Appl Eng Agric. 2014;30(2):199-204.

St-Pierre NR, Weiss WP. Partitioning variation in nutrient composition data of common feeds and mixed diets on commercial dairy farms. J Dairy Sci. 2015;98(7):5004-15.

Undersander D, Shaver R, Linn J, Hoffman P, Peterson $P$. Sampling hay, silage and total mixed rations for analysis. Madison, WI, USA: Cooperative Extension of the University of Wisconsin; 2005.

Van Soest PJ, Robertson JB, Lewis BA. Methods for dietary fiber, neutral detergent fiber, and nonstarch polysaccharides in relation to animal nutrition. J Dairy Sci. 1991;74(10):3583-97.

Weirich Neto PH, Garbuio PW, Souza NM, Delalibera HC, Leitão K. Fragment size of corn silage according to the dry matter and forage harvester adjustments. Eng Agric. 2013;33(4):764-71.

Weiss B, Zhang P, Goeser J, St-Pierre N. TMR sampling: valuable exercise or a random number generator? Proceedings Tri-State Dairy Nutrition Conference; 18-20 abr 2016; Fort Wayne, IN. Fort Wayne: TSDNC; 2016. p. 137-47.

Welch JG. Physical parameters of fiber affecting passage from the rumen. J Dairy Sci. 1986;69(10):2750-4. 